\title{
Coherent patterns and self-focusing of electrons by a thin nonlinear barrier
}

\author{
O. M. Bulashenko \\ Dept. Física Fonamental, Universitat de Barcelona, Diagonal 647, E-08028 Barcelona, Spain. \\ V. A. KochelaP \\ Institute of Semiconductor Physics, National Academy of Sciences, Kiev 252028, Ukraine \\ L. L. Bonilla \\ Universidad Carlos III de Madrid, Butarque 15, E-28911 Leganés, Spain
}

(Received 15 July 1996)

\begin{abstract}
Electron transport across a planar nonlinear barrier where the potential depends selfconsistently on the wave function has been studied. When the amplitude of the incident wave exceeds a certain threshold, a soliton-shaped brightening (darkening) appears on the barrier causing diffraction of the wave. Thus the spontaneously formed transverse pattern can be viewed as a self-induced nonlinear quantum screen. It is shown that the nonlinearity can cause self-focusing of the incident wave into a 'beam', splitting into two 'beams', single or double traces with suppressed reflection or transmission, etc.
\end{abstract}

(C) 1998 Academic Press Limited

Key words: tunnelling, semimagnetic semiconductor heterostructure, $\mathrm{CdTe} / \mathrm{Cd}_{1-x} \mathrm{Mn}_{x} \mathrm{Te}$, spontaneous pattern formation.

\section{Introduction}

Coherent processes in semiconductor nanostructures have attracted considerable interest in recent years. In this contribution, we predict a new nonlinear phenomenon: spontaneous formation of coherent spatial patterns in semiconductor heterostructures.

As an example we consider carrier transport across a quantum nonlinear barrier which is formed by a semimagnetic material $\mathrm{Cd}_{1-y} \mathrm{Mn}_{y} \mathrm{Te}$ embedded in CdTe. For this particular case, the nonlinearity is caused by the exchange interaction between the carrier spin and the magnetic impurities (i.e. Mn inside the barrier). The exchange interaction, treated in a mean-field approximation, gives rise to an effective attractive oneparticle potential inside the barrier (the magnetic polaron effect [1, 2]). In contrast to previous studies which were restricted to one-dimensional situations when longitudinal and transverse degrees of freedom were assumed to be decoupled [3, 4], we consider a fully multidimensional tunneling model. We show that the additional space dimensions open up the possibility for spontaneous pattern formation in transverse directions.

\section{Nonlinear Schrödinger equation}

Considering a thin barrier in the $x y$-plane and modeling its potential as a $\delta$-function one can simplify 
greatly the calculations without modifying qualitatively the results. As we shall see, patterns may form on the barrier and then diffract the incident beam. By analogy with optics, we may consider the barrier as a self-induced screen. The steady-state scattering problem in a mean-field approximation can be traced to a nonlinear Schrödinger equation in the form $[2,3]$

$$
-\frac{\hbar^{2}}{2 m} \Delta \psi(\boldsymbol{r})+\left(V_{0}-A|\psi(\boldsymbol{r})|^{2}\right) \delta(z) \psi(\boldsymbol{r})=E \psi(\boldsymbol{r}) .
$$

Here $V_{0}=V_{b}+V_{m}$, where $V_{b}$ is the barrier height related to the concentration of Mn, and the potential $V_{m}$ is produced by the external magnetic field $B_{0}\left(V_{m} \propto B_{0}\right)$ [2]. The self-consistent potential induced by exchange field is proportional to the probability $|\psi(\boldsymbol{r})|^{2}$ of the carrier being located at $\boldsymbol{r}$ with the coefficient $A$. The expressions for $V_{m}$ and $A$ can be found in [3].

We seek the solution in the form

$$
\psi(x, y, z)=\left\{\begin{array}{lr}
a e^{i k z}+b(x, y, z) e^{-i k z}, & z<0 \\
c(x, y, z) e^{i k z}, & z>0
\end{array}\right.
$$

where the amplitude $a$ of the incident wave is fixed (real) and the carrier energy $E=\frac{\hbar^{2} k^{2}}{2 m}$. We assume that there is no current inflow along the screen (the only inflow into the system is from $z=-\infty$ ). Thus only those solutions satisfying the condition of zero inflow at $z=0, x, y \rightarrow \pm \infty$ will be considered. It is convenient to introduce dimensionless quantities by means of the definitions $\tilde{x}=\sqrt{2} k x, \tilde{y}=\sqrt{2} k y, \tilde{z}=k z, \tilde{b}=b / a$, $\tilde{c}=c / a, \alpha=m V_{0} /\left(\hbar^{2} k\right)$, and $\beta=m A a^{2} /\left(\hbar^{2} k\right)$. Insertion of eqn (2) into eqn (1) for $z \neq 0$ yields

$$
\begin{aligned}
\Delta_{\perp} \tilde{b}+\frac{1}{2} \partial_{\tilde{z} \tilde{z}} \tilde{b}-i \partial_{\tilde{z}} \tilde{b}=0, & \tilde{z}<0 \\
\Delta_{\perp} \tilde{c}+\frac{1}{2} \partial_{\tilde{z} \tilde{z} \tilde{c}+i \partial_{\tilde{z}} \tilde{c}=0,} & \tilde{z}>0 .
\end{aligned}
$$

By using the continuity of the wavefunction $\psi$, one gets, at $z=0$

$$
\partial_{\tilde{z}} \tilde{c}-\partial_{\tilde{z}} \tilde{b}+2 i(\tilde{c}-1)=2\left(\alpha-\beta|\tilde{c}|^{2}\right) \tilde{c} .
$$

Equations (3) and (4) have spatially uniform solutions $\tilde{c}=\xi+i \zeta$ such that $\zeta=\beta \xi^{2}-\alpha \xi,|\tilde{c}|^{2}=\xi$, and

$$
\beta^{2} \xi^{3}-2 \alpha \beta \xi^{2}+\left(\alpha^{2}+1\right) \xi-1=0 .
$$

A straightforward analysis of this equation demonstrates that there is only one real root for $\alpha<\sqrt{3}$ and there are three real roots under the conditions: $\alpha>\sqrt{3}$, and $\beta^{-}<\beta<\beta^{+}$with $\beta^{\mp}=\frac{2}{27}\left[\mp\left(\alpha^{2}-3\right)^{3 / 2}-\alpha^{3}-9 \alpha\right]$. Thus multiple solutions for the transmitted wave can be achieved at the threshold value $\alpha_{0}=m V_{0} / k \hbar^{2}=\sqrt{3}$ by varying the barrier height, the external magnetic field $B_{0}$ and/or the energy of the incident wave. Since $\beta \propto a^{2}$, multiple solutions exist within a certain interval of incident wave amplitudes for any strength of the nonlinearity $A$.

\section{Weakly overcritical regime}

Three uniform solutions coalesce at the tricritical parameter values: $\alpha_{0}=\sqrt{3}, \beta_{0}=-8 \sqrt{3} / 9$, $\xi_{0}=$ $3 / 4, \zeta_{0}=-\sqrt{3} / 4$. We look for solutions near the tricritical point $\alpha=\alpha_{0}+\delta, \beta=\beta_{0}-\gamma$, where $\delta$ and $\gamma$ are small. This consideration corresponds to weakly nonuniform solutions $\tilde{c}=\xi_{0}+\xi_{1}(\tilde{x}, \tilde{y})+i\left\{\zeta_{0}+\zeta_{1}(\tilde{x}, \tilde{y})\right\}$ with $\xi_{1} \ll \xi_{0}, \zeta_{1} \ll \zeta_{0}$. Substituting this ansatz into eqns(3) and (4) we obtain the following simple amplitude equations

$$
\begin{aligned}
\partial_{\tilde{x} \tilde{x}} \xi_{1}+\partial_{\tilde{y} \tilde{y}} \xi_{1} & =\frac{\delta}{\sqrt{3}} \xi_{1}-\frac{32}{27} \xi_{1}^{3}+\frac{\sqrt{3}}{4}\left(\frac{3}{4} \gamma-\delta\right)+O\left(\delta^{5 / 2}\right), \\
\zeta_{1} & =\xi_{1} / \sqrt{3}+O(\delta) .
\end{aligned}
$$


We note that our solutions vary on a large spatial scale $\tilde{x}=\sqrt{2} k x=O\left(\delta^{-1 / 2}\right) \gg 1$, so that the typical transverse length of the solutions is much larger than the wavelength $1 / k$.

With the substitutions: $\xi_{1}=\frac{3}{4} 3^{1 / 4} \delta^{1 / 2} u, \tilde{x}=3^{1 / 4} \delta^{-1 / 2} X, \tilde{y}=3^{1 / 4} \delta^{-1 / 2} Y$, eqn (6) can be written in the simpler form: $\partial_{X X} u+\partial_{Y Y} u=u-2 u^{3}+\mu+O(\delta), \mu=3^{-1 / 4} \delta^{-3 / 2}\left(\frac{3}{4} \gamma-\delta\right)=O(1)$. We report here only the results for $\gamma=\frac{4}{3} \delta$ (corresponding to the most symmetrical situation $\mu=0$ ) and $y$-independent solutions. By doing so, explicit formulae can be easily obtained. If $u=u(X)$ (two-dimensional solutions of the full problem depending only on one transversal coordinate), the parameter-free equation $\partial_{X X} u=u-2 u^{3}$ can be integrated once, yielding the result $\left(\partial_{X} u\right)^{2}=u^{2}-u^{4}+C$. This equation admits nonuniform solutions satisfying the condition of zero flux as $X \rightarrow \pm \infty$ only if $C=0$. In this case we obtain the solutions $u=\eta \operatorname{sech}\left(X-X_{0}\right)$, with $\eta=1$ for the soliton and $\eta=-1$ for the antisoliton. Next, we choose $X_{0}=0$, use relation (6) and obtain the transmitted and reflected amplitudes on the screen:

$$
\begin{aligned}
& \tilde{c}(\tilde{x})=\frac{\sqrt{3}}{2} e^{-i \pi / 6}\left[1+\sqrt{3} \eta \lambda \operatorname{sech}(\lambda \tilde{x}) e^{i \pi / 3}\right], \\
& \tilde{b}(\tilde{x})=-\frac{1}{2} e^{i \pi / 3}\left[1-3 \sqrt{3} \eta \lambda \operatorname{sech}(\lambda \tilde{x}) e^{-i \pi / 6}\right],
\end{aligned}
$$

where $\lambda=3^{-1 / 4} \delta^{1 / 2}$. We have checked that the solutions are linearly stable when we consider the timeevolution problem subject to the boundary conditions discussed earlier.

The amplitudes of the transmitted and reflected waves outside the screen can be found from eqn (3), using the boundary conditions at $z=0$ and ignoring the small terms $\partial_{\tilde{z} \tilde{z}} \tilde{c}$ and $\partial_{\tilde{z} \tilde{z}} \tilde{b}$. In our two-dimensional problem the intensities of the reflected and transmitted waves are nonuniform in space in contrast to the one-dimensional problem where they are constant. Denoting $|\tilde{c}(\tilde{x}, \tilde{z})|^{2}=\Psi_{t}^{0}\left\{1+\Psi_{t}(\tilde{x}, \tilde{z})\right\},|\tilde{b}(\tilde{x}, \tilde{z})|^{2}=\Psi_{r}^{0}\left\{1+\Psi_{r}(\tilde{x}, \tilde{z})\right\}$, and using the soliton-type solutions (7), we obtain $\Psi_{t}^{0}=3 / 4, \Psi_{r}^{0}=1 / 4$. The nonuniform parts of the intensities are given by

$$
\Psi_{r, t}(\tilde{x}, \tilde{z})=\eta \frac{\sigma_{r, t} \lambda}{\sqrt{\pi|\tilde{z}|}} \int_{-\infty}^{\infty} \cos \left[\frac{\left(\tilde{x}-\tilde{x}^{\prime}\right)^{2}}{4|\tilde{z}|}+\phi_{r, t}\right] \operatorname{sech}\left(\lambda \tilde{x}^{\prime}\right) d \tilde{x}^{\prime}
$$

where $\sigma_{r}=-3, \sigma_{t}=\sqrt{3}$, and the phase $\phi$ in the argument of cosine is given by $\phi_{t}=\frac{1}{12} \pi$, and $\phi_{r}=-\frac{5}{12} \pi$.

Spatial distributions of the wave intensities are obtained by numerical integration of eqn (8) and presented in Fig. 1 in terms of the scaled coordinates $X=\lambda \tilde{x}, Z=4 \lambda^{2} \tilde{z}$. The off-screen wave intensities are shown starting from certain nonzero values of $Z$. The wave intensity on the screen is shown as a thin strip in the middle of Fig. 1. The results can be interpreted as follows.

When the amplitude of the incident wave (carrier flux) exceeds a certain threshold, the carriers spontaneously induce polarization of the magnetic ions in the barrier (magnetopolaronic effect) corresponding to the soliton (antisoliton) and, then, diffract on it due to the nonlinear feedback. In particular, for the soliton solution $(\eta=1)$ we observe local self-brightening of the transmitted wave with simultaneous local suppression of the reflected wave. The transmitted wave is focused into a 'beam' of higher intensity with a maximum outside the screen at $Z \approx 1.7$ (Fig. 1). However, for the antisoliton solution $(\eta=-1)$ the maxima and minima are interchanged (with respect to the soliton solution), so that the transmitted wave is split in two 'beams', with a suppressed trace between them. The type of solution (i.e., self-brightening or self-darkening of the transmission) realized in practice depends on additional conditions such as: type of the imperfections pinning the soliton, boundary conditions, past history, and so on.

Note that while we have considered, as an example, the semimagnetic quantum barrier, the results obtained are quite general and could be applicable to heterostructures with other mechanisms of the nonlinearity (electron-phonon coupling, electron-electron interaction, etc.). For instance, electron-electron interactions result in a repulsive potential $(A>0)$ [5]. For that case, a similar derivation can be carried out, but instead of the barrier, multistability of uniform solutions appears for the quantum well $\left(V_{0}<0\right)$, when $\alpha<-\sqrt{3}$, and the corresponding diffraction pattern will be quite different (see [6]). 


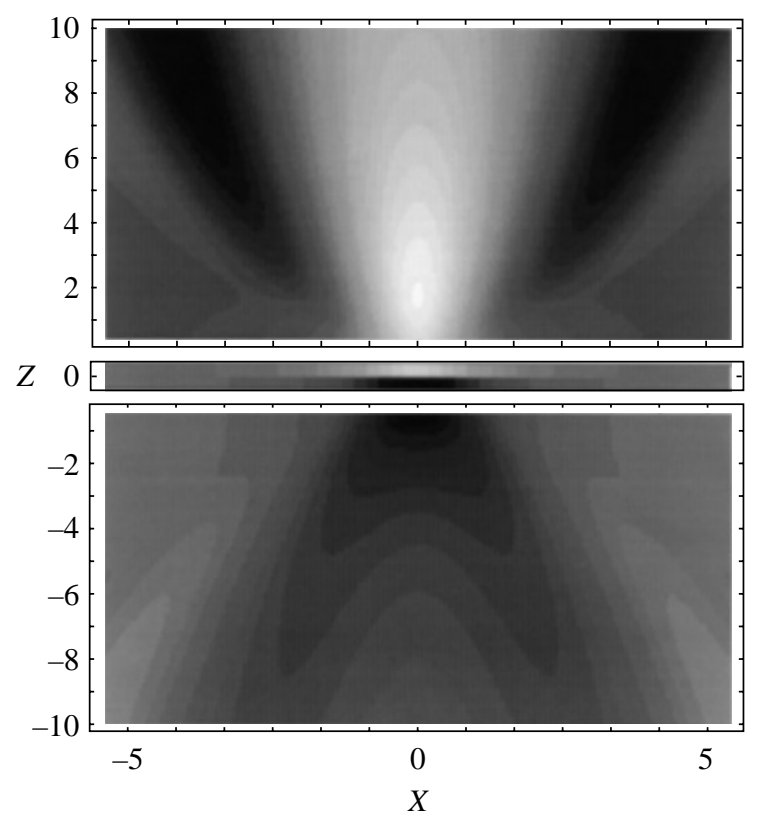

Fig. 1. Density plots for wavefunction intensities corresponding to scattering by the self-induced nonuniform pattern on the screen at $Z=0$. White (black) regions correspond to the maximum (minimum) of the intensity for the soliton solution on the screen $(\eta=1)$, and vice versa for the antisoliton solution $(\eta=-1)$.

In conclusion, spontaneous formation of spatial transverse patterns, which are quantum-mechanically coherent, is expected to occur in semiconductor heterostructures with a thin nonlinear barrier. Self-diffraction of the carrier wave on the transverse patterns gives rise to interesting phenomena like self-brightening or darkening of the transmitted wave, self-focusing, etc.

Acknowledgements - OMB acknowledges support by the Generalitat de Catalunya, Spain. This work has been supported by the DGICYT grants PB92-0248 and PB94-0375, and by the EU Human Capital and Mobility Programme contract ERBCHRXCT930413.

\section{References}

[1] P. A. Wolff, in Semiconductors and Semimetals Vol. 25, Diluted Magnetic Semiconductors, edited by J. K. Furdyna and J. Kossut, Academic Press, New York (1988), Chap. 10 .

[2] J. W. Wu, A. V. Nurmikko, and J. J. Quinn, Solid State Comm. 57, 853-856 (1986).

[3] P. Hawrylak, M. Grabowski, and J. J. Quinn, Phys. Rev. B44, 13082-13084 (1991).

[4] B. A. Malomed and M. Ya. Azbel, Phys. Rev. B47, 10402-10406 (1993).

[5] G. Jona-Lasinio, C. Presilla, and F. Capasso, Phys. Rev. Lett. 68, 2269-2272 (1992).

[6] O. M. Bulashenko, V. A. Kochelap, and L. L. Bonilla, Phys. Rev. B54, 1537-1540 (1996). 